SCIENTIFIC REPORT

\title{
Clinical evaluation of the pressure phosphene tonometer in patients with glaucoma
}

\author{
E Rietveld, D A van den Bremer, H J Völker-Dieben
}

Br J Ophthalmol 2005;89:537-539. doi: 10.1136/bjo.2004.050922

Aim: To evaluate the reliability of the pressure phosphene tonometer in comparison with the Goldmann applanation tonometer.

Methods: 45 consecutive patients with glaucoma (78 eyes) participated in the study. Eyes with previous eye surgery, a documented peripheral visual field defect, a refractive error of more than 5 dioptres, and patients who were unable to understand the procedure were excluded from the study. Intraocular pressure was measured with a pressure phosphene tonometer by one examiner and with a Goldmann applanation tonometer by two other examiners no more than 15 minutes apart. A second series of measurements was performed several weeks later on 34 patients (59 eyes). There was no communication between examiners or between examiner and patient regarding test results.

Results: No statistically significant correlation was found between the applanation tonometry values and those obtained with a pressure phosphene tonometer.

Conclusion: The pressure phosphene tonometer is not suitable for reliably measuring intraocular pressure.

n 1998, Fresco described a method for the self measurement of intraocular pressure and compared its results to the "gold standard" Goldmann applanation tonometer (AT). ${ }^{1}$ The pressure phosphene tonometer (PT) is a device that is able to create a phosphene in a patient's eye by gradually increasing the pressure on the eyeball via the uper eyelid.

The word phosphene, derived from the Greek words phos = light and phainein = to show, is used to describe the psychophysical response to a non-physiological stimulus to the eye-that is pressure instead of light. This principle, the perception of light by external pressure on the eyeball, was presumably first described by Alcmaeon of Croton in approximately 600 BC and was later also described by Aristotle, Purkinje, and Von Helmholtz. ${ }^{2}$

We evaluated the reliability of the PT in the hands of an ophthalmic technician, comparing the results with the values obtained by testing the same patients with the gold standard AT. Self tonometry by the patient was part of the original protocol. However, since the results obtained during the first phase showed no clinical relevance, the protocol was terminated.

\section{PATIENTS AND METHODS}

Patients were recruited for the study between February and June of 2002. Initial selection was based on the examination of the data in the clinical records of the patients. The potential participants were then solicited for voluntary participation during their office visit. Inclusion criteria were an age of 18 years or older, a positive history for glaucoma or a positive family history of glaucoma. Eyes with a history of previous eye surgery, a documented peripheral visual field defect, a refraction of more than 5 dioptres, and patients who were unable to understand the procedure were excluded from the study.

A total of 45 subjects participated in the study. Of these, 34 returned for a second series of measurements. The study group consisted of 24 men (53.3\%) and 21 women (46.7\%) with a mean age of 62 years (range 33-91 years).

During the first visit, the measurement was taken from the right eye in 43 subjects and in the left eye in 35 subjects. In 13 patients only one eye was measured because of exclusion criteria applicable to the fellow eye. During the second visit, the measurement was taken from the right eye in 33 subjects and from the left eye in 26 subjects. This means that intraocular pressure (IOP) was measured in a total of 137 instances using the pressure PT and compared to a similar number of measurements with the Goldmann AT.

Following explanation of the procedure, written informed consent for participation in the study was obtained, and the ability of the patient to perceive the phosphene was evaluated. In all cases the initial measurements were done with the PT, before any kind of anaesthesia to the eye. The patient was instructed to look down and sideways with almost closed eyelids. Then the PT was brought into position on the nasal side of the upper eyelid and the pressure was gradually increased. If the phosphene could not be perceived, it was usually possible to stimulate the appearance of the phosphene by slowly moving the PT up and down over the medial part of the eyeball. After the ability to recognise the phosphene was confirmed the patient was included in the study.

The first series of measurements was performed in 45 consecutive glaucoma patients ( 78 eyes). The study measurements were performed as follows. The pressure of the PT against the upper eyelid was gradually increased by the ophthalmic technician until the patient indicated the perception of a phosphene after which the pressure value indicated by the PT was read and recorded. The measurement was performed three times on one or both eyes at intervals of approximately 10-20 seconds. The mean of those three PT values was used in the study. The patients were not informed about the values obtained with the pressure PT since it was a new device under investigation.

Following this procedure one of the ophthalmologists measured the intraocular pressure in both eyes using a calibrated AT. The time between PT and AT measurements varied from 5 minutes to 15 minutes. The ophthalmologist was not aware of the previous results. All measurements were performed in the morning.

A second series of measurements following the same protocol was performed in 34 patients ( 59 eyes) after a period of $1-8$ weeks, this time in the afternoon.

Abbreviations: AT, applanation tonometer; PT, phosphene tonometer 


\begin{tabular}{llllll}
\hline \multicolumn{1}{l}{ Table 1 } & First visit & & & \\
\hline & No & Minimum & Maximum & Mean & SD \\
\hline Right eye & & 10 & 40 & 19.3 & 4.9 \\
AT & 43 & 10 & 27 & 15.9 & 2.9 \\
PT & 43 & & 45 & 18.8 & 5.9 \\
Left eye & 35 & 11 & 30 & 16.7 & 4.0 \\
AT & 35 & 10 & & & \\
PT & & & & \\
\hline
\end{tabular}

Table 2 Second visit

\begin{tabular}{llllll}
\hline & No & Minimum & Maximum & Mean & SD \\
\hline Right eye & & & & & \\
AT & 33 & 14 & 20 & 20.9 & 4.9 \\
PT & 33 & 12 & 33 & 15.1 & 2.0 \\
Left eye & 26 & 14 & 25 & 15.0 & 5.5 \\
AT & 26 & 12 & & & \\
PT & & & & \\
\hline
\end{tabular}

Table 3 Pooled measurements

\begin{tabular}{llllll}
\hline & No & Minimum & Maximum & Mean & SD \\
\hline AT & 137 & 10 & 45 & 19.9 & 5.3 \\
PT & 137 & 10 & 30 & 15.7 & 3.0 \\
AT-PT & 137 & -9 & 30 & 4.2 & 6.3 \\
\hline
\end{tabular}

The pressure PT was made available by Bausch and Lomb. The action of the PT is bases on a calibrated spring attached to a flat circular probe, with a diameter equal to the Goldmann type applanation tonometer. The scale on the PT is divided into $2 \mathrm{~mm} \mathrm{Hg}$ units and runs from 10 to 40 . The PT indicator remains at the position of the highest value measured.

Mean values, standard deviations, and correlation coefficients were calculated using SPSS v9.0 statistical software.

The institutional review board ethics committee approval was not required for this study.

\section{RESULTS}

During the first visit, the mean value measured with AT in the right eye was $19.3 \mathrm{~mm} \mathrm{Hg}$ with a standard deviation (SD) of $4.9(\mathrm{n}=43$; minimum $=10$, maximum $=40)$. The mean value with PT was 15.9 (SD 2.9) $\mathrm{mm} \mathrm{Hg}$ ( $\mathrm{n}=43$; minimum $=10$, maximum $=27$ ). The mean value measured with AT in the left eye was 18.8 ( $\mathrm{SD} 5.9) \mathrm{mm} \mathrm{Hg}(\mathrm{n}=35$; minimum = 11 , maximum $=45$ ). The mean value with PT was 16.7 (SD 4.0) $\mathrm{mm} \mathrm{Hg}(\mathrm{n}=35$; minimum $=10$, maximum = 30) (table 1). During the second visit, the mean value measured with AT was 21.0 ( SD 5.5) $\mathrm{mm} \mathrm{Hg}(\mathrm{n}=26$; minimum $=14$, maximum $=33$ ). The mean value with PT was 15.0 (SD 2.6) $\mathrm{mm} \mathrm{Hg}(\mathrm{n}=26$; minimum $=12$, maximum $=25)$, and the mean value measured with AT was 20.9 (SD 4.9) $\mathrm{mm} \mathrm{Hg}$ $(\mathrm{n}=33$; minimum $=14$, maximum $=32)$. The mean value measured with PT was 15.1 (SD 2.0) $\mathrm{mmHg}$ ( $\mathrm{n}=33$; minimum $=12$, maximum $=20$ ) (table 2$)$.

If we focus exclusively on the eyes-that is, the 137 times that an eye was measured with a PT and the value was compared with the value measured with the AT, we arrive at the following findings: The mean IOP measured with the AT was 19.9 (SD 5.3) $\mathrm{mmHg}(\mathrm{n}=137 ;$ minimum $=10$, maximum $=45)$. The mean IOP measured with the PT was 15.7 ( SD 3.0) $\mathrm{mm} \mathrm{Hg}(\mathrm{n}=137$; minimum = 10, maximum 30) (table 3 ).

Figure 1 shows the intraocular pressures measured with AT values on the $X$ axis plotted against the pressures measured with PT on the Y axis. The correlation calculated using Spearman's rho is -0.141 with a significance of 0.100 . When plotting the values of the intraocular pressure measured with the applanation tonometer against the difference between the AT and PT values (fig 2), there is a good linear correlation (correlation coefficient 0.877 ) between the difference

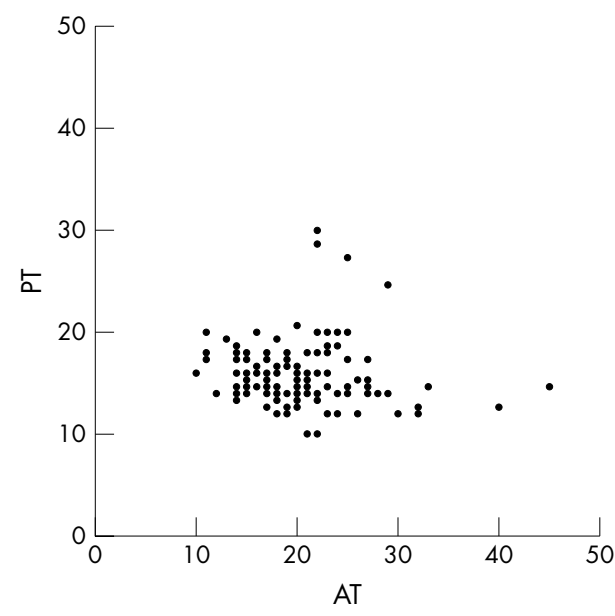

Figure 1 Correlation between the values obtained with the applanation tonometer (AT) and a pressure phosphene tonometer (PT). The values are expressed in $\mathrm{mm} \mathrm{Hg}$. 


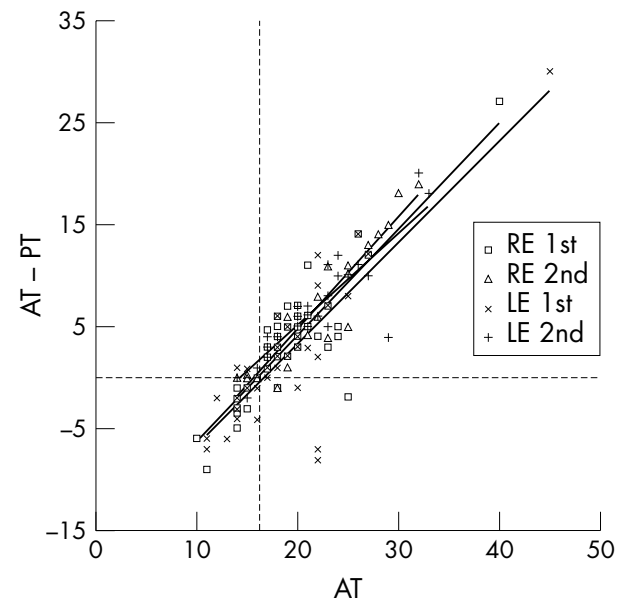

Figure 2 Correlation between the applanation tonometer values (AT) and the difference between the values obtained with the pressure phosphene tonometer and the applanation tonometer for each group (AT-PT). The values are expressed in $\mathrm{mm} \mathrm{Hg}$.

between applanation and pressure phosphene tonometry measurements and the AT values. The results for the separate groups-that is, only right eyes, only left eyes at the first or the second visit consistently showed the same pattern as the overall results.

\section{DISCUSSION}

The results concerning the main issue, "To what extent are the intraocular pressures measured with the pressure phosphene tonometer comparable with those measured with the Goldmann tonometer?" are much less favourable with regard to the practical applicability and reliability of the pressure phosphene tonometer, than the results presented by Fresco. ${ }^{1}$ He observed a mean IOP of $15.5 \mathrm{~mm} \mathrm{Hg}$ using the applanation tonometer and a mean IOP of $15.2 \mathrm{~mm} \mathrm{Hg}$ using the pressure phosphene tonometer, a difference of only $0.3 \mathrm{~mm} \mathrm{Hg}$.

We observed a considerably larger difference between the measurements provided by the two techniques in our group of patients. The mean IOP measured with the applanation tonometer was $19.9 \mathrm{~mm} \mathrm{Hg}$ and the mean IOP measured with the pressure phosphene tonometer was $15.7 \mathrm{~mm} \mathrm{Hg}$. The difference between those means is considerably larger than can be attributed to interobserver or intraobserver variation. ${ }^{3}$ The results of our study do not confirm the correlation coefficient of 0.7 in Fresco's publication.
Figure 2 clearly demonstrates that the more the applanation tonometry value exceeds $16 \mathrm{~mm} \mathrm{Hg}$, the larger also the difference between AT value and PT value becomes. A regression line through these points intersects the line of zero difference at about $16 \mathrm{~mm} \mathrm{Hg}$. This suggests that a measurement with the phosphene tonometer yields random values around $16 \mathrm{~mm} \mathrm{Hg}$ with no relevance to the actual intraocular pressure as measured by applanation tonometry.

In a previous pilot study we found that there was a good correlation between the PT values obtained by a technician and the PT values resulting from self tonometry by the patient. The PT tonometry by the technician however showed a considerably shorter learning curve for the patient.

The pressure point used to stimulate the appearance of a phosphene is the same as the location described by Fresco. ${ }^{1}$ The pressure point corresponds to the inferotemporal visual field. That fact is convenient for our purpose, for two reasons. The first reason is that a phosphene can be stimulated most rapidly here. The second reason is that the inferotemporal visual field is usually the final area to become affected by glaucomatous damage.

We conclude that the pressure phosphene tonometer cannot be considered a reliable alternative for Goldmann's applanation tonometry. We did not succeed in finding a relation between the moment of perception of a phosphene and the intraocular pressure.

\section{Authors' affiliations}

E Rietveld, D A van den Bremer, H J Völker-Dieben, Department of Ophthalmology, VU University Medical Center, Amsterdam, Netherlands

Financial support: $€ 250$, grant from the Dutch Glaucoma Society.

Competing interests: none declared

Correspondence to: Eelco Rietveld, MD, Department of Ophthalmology, VU University Medical Center, PO Box 7057, 1007 MB Amsterdam, Netherlands; e.rietveld@vumc.nl

Accepted for publication 1 September 2004

\section{REFERENCES}

1 Fresco BB. A new tonometer-the pressure phosphene tonometer: clinical comparison with Goldman tonometry. Ophthalmology 1998;105:2123-6.

2 Grusser OJ, Grusser-Cornehls U, Hagner M, et al. Purkyne's description of pressure phosphenes and modern neurophysiological studies on the generation of phosphenes by eyeball deformation. Physiologia Bohemoslovaca 1989;38:289-309.

3 Dielemans I, Vingerling JR, Hofman A, et al. Reliability of intraocular pressure measurement with the Goldmann applanation tonometer in epidemiological studies. Graefes Arch Clin Exp Ophthalmol 1994;232:141-4. 